Kyoko Takiguchi · Kohji Itoh • Michie Shimmoto

Pinar T Ozand • Hirofumi Doi • Hitoshi Sakuraba

\title{
Structural and functional study of K453E mutant protective protein/ cathepsin A causing the late infantile form of galactosialidosis
}

\begin{abstract}
To clarify the molecular basis of the late infantile form of galactosialidosis, we characterized a defective protective protein/cathepsin A (PPCA) gene product with the K453E mutation newly found in an Arabic patient with this disease. Immunocytochemical, expression, and metabolic studies revealed that the precursor PPCA was synthesized but not processed to the mature form, and it was degraded in the mutant. A structural model of the mutant PPCA was constructed by amino acid substitution of ${ }^{453}$ glutamic acid for lysine in the crystal structure of the wild type PPCA precursor reported. The results show that the $\mathrm{K} 453 \mathrm{E}$ mutation is located at the dimer interface of the PPCA and reduces the hydrogen bond formation in the dimer. This structural change may cause instability of the PPCA dimer.
\end{abstract}

Key words Protective protein/cathepsin A - Galactosialidosis - Structural modeling - Gene mutation $\cdot \beta$-Galactosidase $\cdot$ Neuraminidase

\section{Introduction}

Lysosomal protective protein/cathepsin A (PPCA; EC 3.2.1.14) is a serine carboxypeptidase that forms a high molecular weight complex with $\beta$-galactosidase $(\mathrm{EC}$

K. Takiguchi $\cdot$ H. Doi

Fujitsu Laboratories Ltd. Chiba, Japan

K. Itoh $\cdot$ M. Shimmoto $\cdot H$. Sakuraba $(\bowtie)$

Department of Clinical Genetics, The Tokyo Metropolitan Institute of Medical Science, Tokyo Metropolitan Organization for Medical Research, 3-18-22 Honkomagome, Bunkyo-ku, Tokyo 113-8613, Japan

Tel. +81-3-823-2101; Fax +81-3-823-6008

e-mail: sakuraba@rinshoken.or.jp

P.T. Ozand

Departments of Pediatrics, and Biological and Medical Research,

King Faisal Specialist Hospital, Riyadh, Saudi Arabia
3.2.1.23) and neuraminidase (EC 3.2.1.18), and it stabilizes the former enzyme and activates the latter one in lysosomes (protective function). PPCA is synthesized as a $54-\mathrm{kDa}$ inactive precursor. The precursor undergoes dimerization at neutral $\mathrm{pH}$ and is then transported to an acidic compartment. Then it is further processed to a mature active form comprising $32-\mathrm{kDa}$ and $20-\mathrm{kDa}$ subunits held together by disulfide bridges (d'Azzo et al. 1982; Galjart et al. 1988; Bonten et al. 1996).

A genetic defect of PPCA causes an autosomal recessive inherited metabolic disorder, galactosialidosis, associated with a combined deficiency of cathepsin A, $\beta$-galactosidase, and neuraminidase (reviewed by d'Azzo et al. 1995). The clinical features are heterogeneous, and include coarse facies, macular cherry-red spots, skeletal dysplasia, visceromegaly, and neurologic disorders, including cerebellar ataxia and myoclonus. Based on the onset and severity of the disease, galactosialidosis is clinically divided into three subtypes: the early infantile, late infantile, and juvenile/ adult forms. Most patients with the juvenile/adult form of galactosialidosis are of Japanese origin, and they have a common $\mathrm{a}^{+3}$ to $g$ substitution at the exon $7 /$ intron 7 junction (SpDEx7) (Shimmoto et al. 1990). The mutation results in a splicing defect and a decreased amount of PPCA mRNA. On the other hand, patients with the early infantile form of galactosialidosis are of panethnic origin, and a variety of missense mutations causing a deficiency of the mature PPCA have been reported (Shimmoto et al. 1993; Zhou et al. 1996). As to the late infantile form of galactosialidosis, only two kinds of missense mutations, reflecting mild phenotype (Y249N and F440V; the numbering is based on the initiation codon being taken as codon no. 1), have been reported so far (Shimmoto et al. 1993; Zhou et al. 1991, 1996).

In this study, we performed molecular characterization of a defective PPCA with a K453E mutation, which has been newly found in an Arabic patient with the late infantile form of galactosialidosis, and compared the results with those for the Y249N mutant found in Caucasian patients exhibiting a milder clinical phenotype than that. 


\section{Subjects and methods}

Patient

The patient was a 7-year-old Arabic girl. She was born after an unremarkable pregnancy, labor, and delivery. She was noted to have coarse facies at birth. However, no detailed data were available until she was age 7 years, when she was noted to have hepatosplenomegaly. She was admitted to a hospital and underwent a detailed examination. Her mental age was estimated to be 4 years. On physical examination, there were no abnormal findings neurologically. But cranial magnetic resonance imaging revealed small increased T2 intensity lesions within the centrum ovale. Macular cherryred spots and vacuolated lymphocytes were observed. A skeletal survey disclosed mild changes of dysostosis multiplex, including a "J" shaped sella and thickening of the vertebrae.

The results of lysosomal enzyme assays showed a combined deficiency of cathepsin A, $\beta$-galactosidase, and neuraminidase activities in the patient, and gene analyses of cDNA and genomic DNA revealed that the patient was homozygous for the A1357 $\rightarrow \mathrm{G}$ transition, causing an amino acid substitution of glutamic acid for lysine (K453E). Northern blot analysis revealed that the patient had a normal amount of PPCA mRNA (data not shown).

\section{Cell culture}

Skin fibroblasts from the patient and normal subjects were cultured in Ham's F-10 medium supplemented with $10 \%$ fetal calf serum and antibiotics at $37^{\circ} \mathrm{C}$ in a humidified incubator flushed with a $5 \% \mathrm{CO}_{2}-95 \%$ air mixture.

Fibroblasts from a galactosialidosis patient with a decreased amount of PPCA mRNA were transformed with a simian virus 40-adenovirus recombinant, as previously described (Shimmoto et al. 1993), and the transformed cell line, ASVGS-1, was used for expression and metabolic studies.

\section{Enzyme assays}

The activities of cathepsin $\mathrm{A}$, neuraminidase, and $\beta$ galactosidase in cell extracts were determined using $\mathrm{N}$-carbobenzoxy-L-phenylalanyl-L-leucine or 4-methylumbelliferyl glycosides as the substrate, as previously described (Itoh et al. 1991; Sakuraba et al. 1982). The amount of protein was determined with a dye-binding assay kit (Bio-Rad, Hercules, CA, USA), using bovine serum albumin as a standard.

\section{Immunocytochemistry}

Intracellular PPCA was stained with a rabbit antibody (anti-PP32N12), which had been raised against the aminoterminal oligopeptide of the $32-\mathrm{kDa}$ subunit of human PPCA, as previously described (Satake et al. 1994). This antibody recognizes the 32-kDa subunit of the mature form, and also the precursor form of human PPCA.

Fibroblasts were cultured on a Lab-Tek chamber slide (Nunc, Naperville, IL, USA), and fixed with 2\% (w/v) paraformaldehyde in phosphate-buffered saline (PBS), pH7.4, and then methanol. After the blocking of nonspecific binding with $5 \%(\mathrm{v} / \mathrm{v})$ goat serum and $1 \%(\mathrm{w} / \mathrm{v})$ bovine serum albumin in phosphate-buffered saline (PBS), pH7.4, the cells were reacted with affinity-purified anti-PP32N12 overnight at $4{ }^{\circ} \mathrm{C}$. After being washed with PBS, pH7.4, they were treated with fluorescein isothiocyanate-conjugated $\mathrm{F}$ $(a b ')_{2}$ goat anti-rabbit immunoglobulin G (BioSource, Camarillo, CA, USA).

The stained cells were observed with a confocal laser scanning imaging system (MRC 600; Bio-Rad, Hemel Hempstead, UK) attached to a Nikon microscope (Optiphot 2; Nikon, Tokyo, Japan).

\section{Expression and metabolic studies}

The expression study was performed as previously described (Shimmoto et al. 1993). Briefly, ASVGS-1 cells (Shimmoto et al. 1993) on 10-cm dishes were transfected with the wild-type, or mutant PPCA cDNA having either the K453E or Y249N mutation in an expression vector, pCAGGS (Miyazaki et al. 1989), by the calcium phosphateglycerol shock technique. The cells were harvested after $60 \mathrm{~h}$ and the enzyme activities were determined.

The metabolic study was performed basically according to a previously described method (Shimmoto et al. 1993). ASVGS-1 cells transfected with the plasmid containing the wild-type or a mutant PPCA cDNA were cultured in $6-\mathrm{cm}$ dishes for $36 \mathrm{~h}$, and then labeled for $3 \mathrm{~h}$ with $2 \mathrm{MBq} / \mathrm{ml}$ Expre ${ }^{35} \mathrm{~S}^{35} \mathrm{~S}\left[{ }^{35} \mathrm{~S}\right]$ Protein Labeling Mix (DuPont NEN, Boston, MA, USA). After the culture medium had been changed to non-labelled one, the culture was continued for various periods $(0,3,6$, and $15 \mathrm{~h})$. Then, the cells were harvested and homogenized. The $\left[{ }^{35} \mathrm{~S}\right]$-labeled PPCA was immunoprecipitated with a polyclonal antibody (anticomplex) raised against the high molecular weight complex of PPCA and $\beta$-galactosidase (Nanba et al. 1988), and Protein A-Sepharose (Pharmacia LKB Biotechnology, Uppsala, Sweden). The anti-complex antibody recognizes the purified precursor and the $20-\mathrm{kDa}$ subunit of the mature PPCA and weakly detects the $32-\mathrm{kDa}$ subunit on immunoblotting (Satake et al. 1994). The samples were then subjected to sodium dodecylsulfate-polyacrylamide gel electrophoresis (SDS-PAGE). Radioactive bands were visualized by fluorography.

\section{Structural modeling of mutant PPCA}

The crystal structure of human PPCA was recently determined (Rudenko et al. 1995) and deposited in the Protein Data Bank (PDB; Brookhaven National Laboratory, Upton, NY, USA) (Abola et al. 1997; Sussman et al. 1998), and structural modeling of several kinds of mutant proteins was performed using the data (Rudenko et al. 1998). In this 
study, a model of the K453E mutant protein was constructed by amino acid substitution of ${ }^{453}$ glutamic acid for lysine in the crystal structure of the wild-type PPCA precursor (PDB code, 1IVY) and subsequent energy minimization. All calculations, the model building, and the energy minimization procedures were performed using the molecular modeling software, SYBYL (Tripos, St Louis, MO, USA), installed on a PowerIndigo2 R8000 workstation (Silicon Graphics, Mountain View, CA, USA). The energy minimization was performed on the structure within a $12-\AA$ radius of the replaced residue, using the Tripos force field (Clark et al. 1989) and no charge, for simplicity.

We defined the structure influenced by the amino acid replacement as the residue of the mutant model which differed by more than $0.5 \AA$ from that in the least-square-mean fitted wild-type structure.

\section{Results}

\section{Immunocytochemistry}

The results of immunocytochemical analysis are shown in Fig. 1. Granular inclusions exhibiting strong fluorescence were observed in cultured fibroblasts from a normal subject, which suggested the existence of the mature PPCA in lysosomes. However, granular fluorescence was not detected in cells from the patient with the K453E mutation.

\section{Expression study}

After transient expression in simian virus 40-adenovirustransformed galactosialidosis cells, the K453E mutant exhibited neither cathepsin A activity nor a protective function as to neuraminidase and $\beta$-galactosidase (Table 1). On the other hand, the mutant PPCA with the Y249N mutation, which has been found in the mildest late infantile galactosialidosis patients known (d'Azzo et al. 1995; Shimmoto et al. 1993), and used here as a pathological control, showed residual cathepsin A activity and restoration of the protective function (Table 1).

\section{Metabolic study}

A metabolic study with an anti-complex antibody was performed, and the results are shown in Fig. 2. The antibody recognized the precursor and the $20-\mathrm{kDa}$ subunit of the mature PPCA, but the immunoreaction with the $32-\mathrm{kDa}$ subunit was weak under the assay conditions used here, as described under "Subjects and methods". In addition, there was a nonspecific band around the position of the $32-\mathrm{kDa}$ protein, unfortunately, and thus the nonspecific and specific bands overlapped on the blot and we could not clearly chase the expressed 32-kDa subunit. However, by means of the 54-kDa and 20-kDa bands on SDS-PAGE, we could follow the intracellular processing of the expressed PPCA. After transfection of the wild-type PPCA cDNA, the synthesized
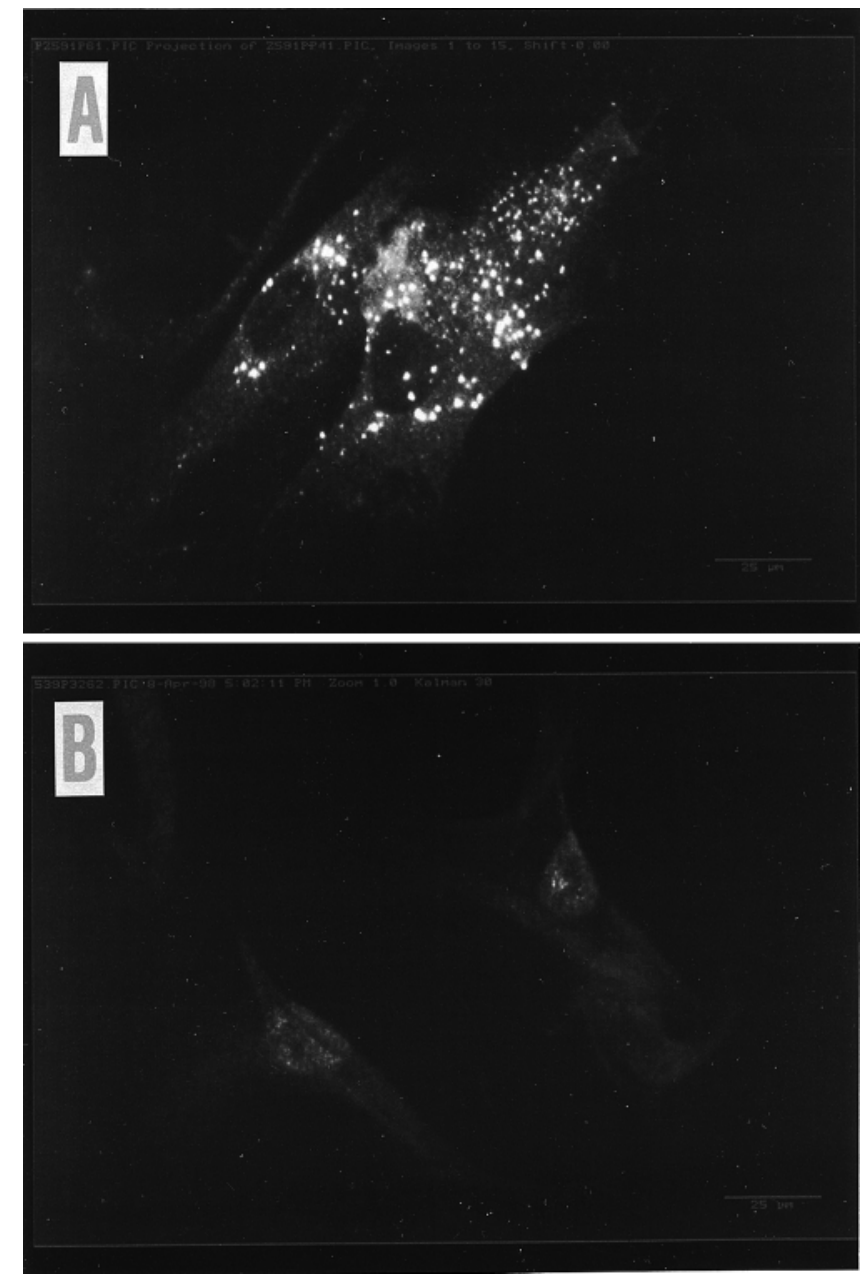

Fig. 1A,B. Immunofluorescence staining of cultured fibroblasts with an anti-PP32N12 antibody; fibroblasts from $\mathbf{A}$ a normal subject, and $\mathbf{B}$ from the patient. Bar, $25 \mu \mathrm{m}$

Table 1. Expression study

\begin{tabular}{llll}
\hline & $\begin{array}{l}\text { Cathepsin A } \\
(\mu \text { mol/h per } \\
\text { mg protein) }\end{array}$ & $\begin{array}{l}\beta \text {-Galactosidase } \\
\text { (nmol/h per } \\
\text { mg protein) }\end{array}$ & $\begin{array}{l}\text { Neuraminidase } \\
(\text { nmol/h per } \\
\text { mg protein) }\end{array}$ \\
\hline Mock & $<0.2$ & 13.0 & 0.6 \\
Wild-type & 20.1 & 80.3 & 21.0 \\
K453E & $<0.2$ & 18.4 & 0.7 \\
Y249N & 2.6 & 73.1 & 12.0 \\
\hline
\end{tabular}

Lysosomal enzyme activities in galactosialidosis fibroblastic cell lines transfected with wild-type and mutant protective protein/carthepsin A (PPCA) cDNAs. Values are means for two independent measurements Mock, Transfected with pCAGGS; wild-type, transfected with the wild-type PPCA cDNA; K453E, transfected with the mutant PPCA cDNA with K453E; Y249N, transfected with the mutant PPCA cDNA with Y249N

precursor was processed rapidly to the mature form. In the K453E mutant, the 54-kDa precursor was synthesized but was not processed to the mature form, and it rapidly disappeared.

On the other hand, the Y249N mutant expressed the 54$\mathrm{kDa}$ precursor, but its processing to the mature form was 
Fig. 2. Metabolic study of the expressed protective protein / cathepsin A (PPCA). ASVGS-1 cells transfected with the plasmid containing the wild-type or the mutant PPCA cDNA were radiolabeled for $3 \mathrm{~h}$ and then chased for the times indicated above the lanes. The labeled PPCA was analyzed by the method described under "Subjects and methods". Mock, Transpected with pCAGGS
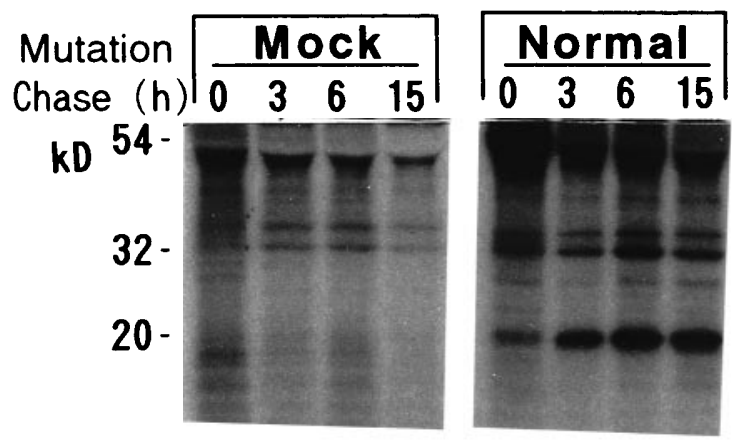
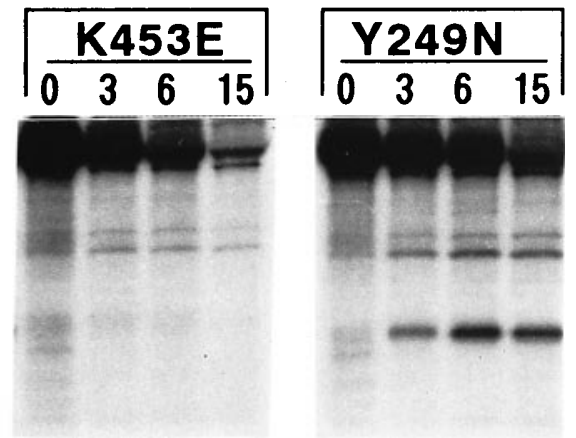

slow, and the intensity of the 20-kDa band was lower than in the case of the wild-type.

\section{Structural modeling}

According to the crystal structure reported by Rudenko et al. (1995), human PPCA consists of "core" and "cap" domains. The core domain contains ten central $\beta$-sheets, and an additional ten $\alpha$-helices and two small $\beta$-sheets occur on both sides of the central $\beta$-sheets. The cap domain is divided into a "helical" subdomain, composed of three $\alpha$ helices, and a "maturation" subdomain, composed of three $\beta$-sheets. The location of the K453E mutation in the monomer PPCA was determined at first. K453 followed $\beta 12$ (residues 447-452; the numbering is based on the initial methionine being taken as no. 1). Human PPCA is known to form a dimer in cultured fibroblasts and tissues, and each monomer comprising the dimer is named the A- or B-chain in the Protein Data Bank (PDB) data. We performed localization of K453E in the three-dimensional structure of the dimer (Fig. 3), and it was found to be located at the dimer interface. As to K453 of the A-chain (A/K453; name of the chain/name of the amino acid residue and codon number), hydrogen bonds were formed between its $\mathrm{H}$-atom and the $\mathrm{O}$-atom of $\mathrm{A} 438$ of the $\mathrm{A}$-chain $(\mathrm{A} / \mathrm{A} 438)$, between its $\mathrm{H}^{\mathrm{\zeta}}$ atom and the O-atom of L277 of the B-chain (B/L277), and between its $\mathrm{H}^{53}$-atom and a water molecule. On the other hand, as to $\mathrm{K} 453$ of the $\mathrm{B}$-chain $(\mathrm{B} / \mathrm{K} 453)$, the hydrogen bonds were formed between its $\mathrm{H}$-atom and the $\mathrm{O}$-atom of $\mathrm{A} 438$ of the B-chain (B/A438), between its $\mathrm{H}^{\mathrm{t} 1}$-atom and the O-atom of L277 of the A-chain (A/L277), and between its $\mathrm{H}^{52}$-atom and the $\mathrm{O}$-atom of $\mathrm{A} 279$ of the A-chain (A/ A279), as shown in Fig. 4. These findings suggest that K453 contributes to the stability of the dimer by forming hydrogen bonds with the atoms of the other monomer.

The substitution of lysine with glutamic acid at codon 453 would abolish the hydrogen bond formation, except for the bond between its $\mathrm{H}$-atom and the $\mathrm{O}$-atom of A410 of the other monomer, suggesting reduction of the stable dimerization because of hydrogen bonds. The residues influenced by the mutation of the A-chain were N276, Y278, A279, and C281, all of which were in the B-chain, and the residues influenced by the mutation of the B-chain were Y278, A279, P280, C281, and I437, all of which were in the A-chain. Except for I437, which was located at $\beta 11$ of the

core domain, all these influenced residues were located in the cap domain.

A structural model of the Y249N mutant protein was also constructed, by means of the method described here, to compare it with the K453E mutant protein. The Y249N mutation is located in the cap domain (Fig. 3). The mutation alters the hydrogen bonding network. With the amino acid substitution of the A-chain, the hydrogen bond between its $\mathrm{H}$-atom and the $\mathrm{O}^{\delta 1}$-atom of $\mathrm{N} 247$ of the A-chain would be kept, but the bond between its $\mathrm{H}^{\mathrm{n}}$-atom and the water molecule would be lost. In the B-chain, the mutation alters the network from the bond between the $\mathrm{OH}$ - of $\mathrm{Y} 249$ and $\mathrm{H}^{111}$ atom of R375 of the B-chain to the bond between the $\mathrm{H}^{821}$ atom of $\mathrm{N} 249$ and $\mathrm{O}^{\delta 1}$-atom of D250 of the B-chain. Q213 of the A-chain was deduced to be influenced by the mutation, which was located in the cap domain (Fig. 5).

\section{Discussion}

The molecular basis of galactosialidosis at the protein level has not yet been clarified. Since the recent construction of a three-dimensional structural model of human PPCA, a new approach for clarifying the molecular pathogenesis of the disease has been developed. Rudenko et al. (1998) modeled 11 amino acid substitutions causing galactosialidosis in the PPCA structure. They have reported that 9 of them $(\mathrm{Q} 49 \mathrm{R}$, S51Y, W65R, S90L, V132M, L236P, Y395C, M406T, and G439S [amino acid numbers cited by Rudenko et al. are those starting from the amino-terminus of the precursor polypeptide deleting the signal peptide sequence. But amino acids have been numbered from the initiation codon in our previous reports, according to the standard method. So, our numbering is used here to identify these substitutions]) are located at positions likely to drastically alter the folding and stability of the mutant protein, and the other 2 (Y249N and F440V), which are associated with residual mature PPCA protein and moderate clinical manifestations, have a milder effect on the protein structure. We mapped the K453E mutation, which was newly found in an Arabic patient with the late infantile form of galactosialidosis, in the structure of the wild-type PPCA and characterized the effect of the amino acid substitution on the structural change in the PPCA dimeric model. 
Fig. 3. Three-dimensional structure of the PPCA precursor dimer. The backbone structure is presented as shaded ribbons with the domains and subdomains in different colors; the core domain of the A-chain is shown in cyan, the core domain of the B-chain in blue, the helical subdomain of the A-chain in red, the helical subdomain of the B-chain in $\mathrm{ma}^{-}$ genta, the maturation subdomain of the A-chain in yellow, and the maturation subdomain of the Bchain in orange. The side-chains of K453 and Y249 are shown in green and white, respectively

Fig. 4. Structural changes caused by the K453E mutation. The residues influenced by the replacement of K453 (blue) with E (red) are shown. The residues of the wild type and the mutant are colored cyan and magenta, respectively. The yellow broken lines represent the hydrogen bonds, and the cyan sphere represents the water molecule
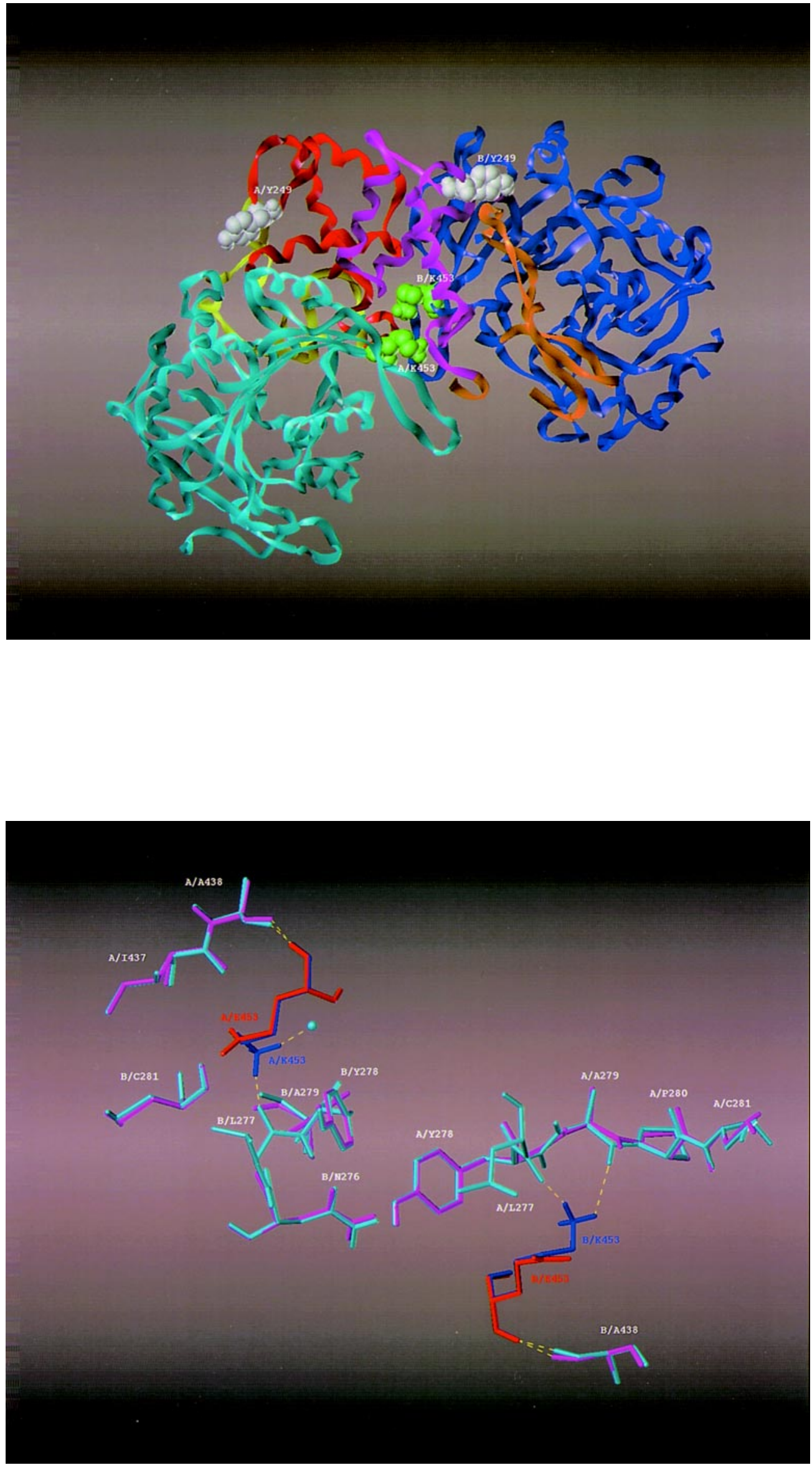
Fig. 5. Structural changes caused by the Y249N mutation. The residues influenced by the replacement of Y249 (blue) with N (red) are shown. The residues of the wild-type and the mutant are colored cyan and magenta, respectively. The yellow broken lines represent the hydrogen bonds, and the cyan sphere represents the water molecule

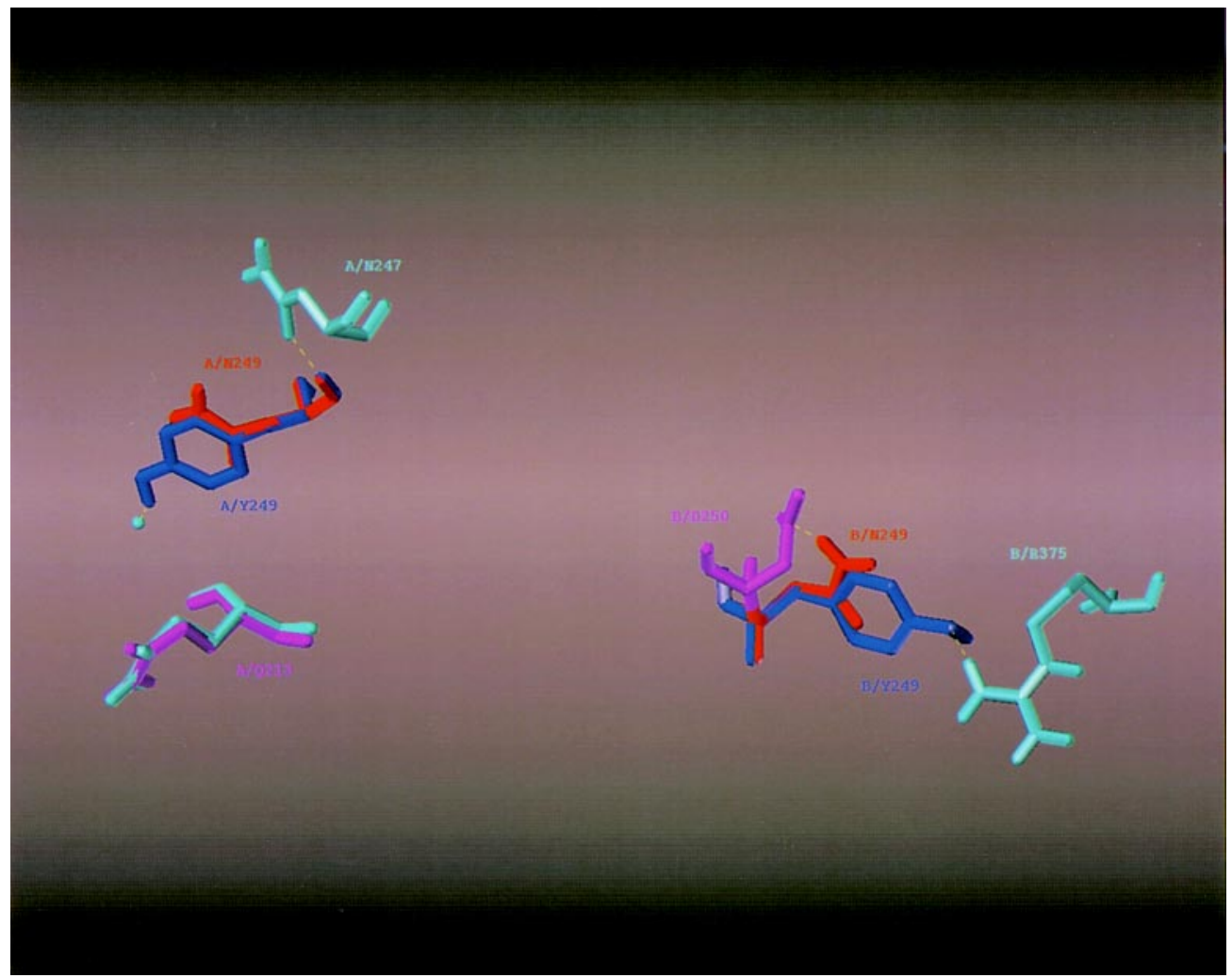

It is predicted that the mutation is located at the dimer interface and reduces the hydrogen bond formation between precursor monomers in the dimeric form. The precursor PPCA folds and dimerizes in the endoplasmic reticulum, and is then proteolytically processed into the mature functional form in lysosomes. Furthermore, the mature PPCA needs to form a high molecular weight complex with $\beta$-galactosidase to exhibit the protective function, and the complex is reconstructed with one $\beta$-galactosidase tetramer and four PPCA dimers (d'Azzo et al. 1995). So, proper dimer formation of PPCA seems to be important for its processing and function. The F440V mutation is located at the dimer interface and its expressed protein is found largely as a monomer and is rapidly degraded in lysosomes (Zhou et al. 1991). The simulation suggests that the K453E replacement would cause destabilization or a functional defect of the dimer. In fact, the biochemical study revealed that, in galactosialidosis cells, the expressed precursor could not be processed to the mature form, and that it was degraded rapidly. Rudenko et al. (1998) predicted that the F440V mutation would cause a hydrophobic cavity, which would result in defective dimerization of the PPCA precursor. So, similar pathophysiological changes may also occur in the K453E mutant.

We also compared the effect of the K453E mutation with that of the Y249N substitution. Within the late infantile group reported, patients carrying the Y249N mutation have a clinically and biochemically milder phenotype than those with the F440V substitution (Zhou et al. 1996). The Y249N mutation was predicted to be located apart from the dimer interface and to have a smaller effect on the hydro- gen bond network of the PPCA dimer than the K453E mutation. This was consistent with the finding that the expressed Y249N gene product was partly processed to the mature form and showed residual cathepsin A activity. In contrast, the enzyme activity of the expressed product with the K453E mutation was much lower. The apparent difference between the K453E mutant and other mutants found in patients with the early infantile form of galactosialidosis could not be detected biochemically under the assay condition used here. But the specific structural change in the K453E mutant may reflect biochemical and clinical features.

In conclusion, we characterized the structural and functional defects caused by the $\mathrm{K} 453 \mathrm{E}$ mutation. The data show that heterogeneous functional changes of PPCA occur in galactosialidosis cells according to the structural changes caused by various gene mutations. Extensive studies of the structural and biochemical aspects will facilitate the further clarification of the complicated molecular pathology of galactosialidosis.

Acknowledgments This work was partly supported by grants from the Tokyo Metropolitan Government, the Ministry of Education, Science, Sports, and Culture of Japan, and the Ministry of Health and Welfare of Japan.

\section{References}

Abola EE, Sussman JL, Prilusky J, Manning NO (1997) Protein data bank archives of three-dimensional macromolecular structures. In: 
Carter CW, Sweet RM (eds) Methods in enzymology, vol. 277. Macromolecular crystallography. Academic, San Diego, pp 556-571

Bonten E, van der Spoel A, Fornerod M, Grosveld G, d'Azzo A (1996) Characterization of human lysosomal neuraminidase defines the molecular basis of the metabolic storage disorder sialidosis. Genes Dev 10:3156-3169

Clark M, Cramer RDIII, van Opdenbosch N (1989) Validation of the general purpose Tripos 5.2 force field. J Comp Chem 10:982-1012

d'Azzo A, Hoogeveen A, Reuser AJ, Robinson D, Galjaard H (1982) Molecular defect in combined beta-galactosidase and neuraminidase deficiency in man, Proc Natl Acad Sci USA 79:4535-4539

d'Azzo A, Andria G, Strisciuglio P, Galjaard H (1995) Galactosialidosis. In: Scriver CR, Beaudet AL, Sly WS, Valle D (eds) The metabolic and molecular bases of inherited disease, 7th edn. McGraw-Hill, New York, pp 2825-2837

Galjart NJ, Gillemans N, Harris A, van der Horst GTJ, Verheijen FW, Galjaard H, d'Azzo A (1988) Expression of cDNA encoding the human "protective protein" associated with lysosomal $\beta$-galactosidase and neuraminidase: homology to yeast proteases. Cell 54:755764

Itoh K, Takiyama N, Nagao Y, Oshima A, Sakuraba H, Potier M, Suzuki Y (1991) Acid carboxypeptidase deficiency in galactosialidosis. Jpn J Hum Genet 36:171-178

Miyazaki J, Takaki S, Araki K, Tashiro F, Tominaga A, Takatsu K, Yamamura K (1989) Efficient selection for high-expression transfectants with a novel eukaryotic vector. Gene 79:269-277

Nanba E, Tsuji A, Omura K, Suzuki Y (1988) Galactosialidosis: direct evidence that a 46-kilodalton protein restores deficient enzyme activities in fibroblasts. Biochem Biophys Res Commun 152:794800

Rudenko G, Bonten E, d'Azzo A, Hol WGJ (1995) Three-dimensional strucure of the human "protective protein": structure of the pre- cursor form suggests a complex activation mechanism. Structure 3:1249-1259

Rudenko G, Bonten E, Hol WGJ, d'Azzo A (1998) The atomic model of the human protective protein/cathepsin A suggests a structural basis for galactosialidosis. Proc Natl Acad Sci USA 95:621-625

Sakuraba H, Aoyagi T, Suzuki Y (1982) Galactosialidosis ( $\beta$ galactosidase and neuraminidase deficiency): a possible role of serine-thiol proteases in the degradation of $\beta$-galactosidase molecules. Clin Chim Acta 125:275-283

Satake A, Itoh K, Shimmoto M, Saido TC, Sakuraba H, Suzuki Y (1994) Distribution of lysosomal protective protein in human tissues. Biochem Biophys Res Commun 205:38-43

Shimmoto M, Takano T, Fukuhara Y, Oshima A, Sakuraba H, Suzuki Y (1990) Japanese-type adult galactosialidosis: a unique and common splice junction mutation causing exon skipping in the protective protein/carboxypeptidase gene. Proc Jpn Acad 66B:217-222

Shimmoto M, Fukuhara Y, Itoh K, Oshima A, Sakuraba H, Suzuki Y (1993) Protective protein gene mutations in galactosialidosis. J Clin Invest 91:2393-2398

Sussman JL, Lin D, Jiang J, Manning NO, Prilusky J, Ritter O, Abola EE (1998) Protein data bank (PDB): database of three-dimensional structural information of biological macromolecules. Acta Crystallogr sect. D, 54:1078-1084

Zhou XY, Galjart NJ, Willemsen R, Gillemans N, Galjaard H, d'Azzo A (1991) A mutation in a mild form of galactosialidosis impairs dimerization of the protective protein and renders it unstable. EMBO J 10:4041-4048

Zhou XY, van der Spoel A, Rottier R, Hale G, Willemsen R, Berry GT, Strisciuglio P, Andria G, d'Azzo A (1996) Molecular and biochemical analysis of protective protein/cathepsin A mutations: correlation with clinical severity in galactosialidosis. Hum Mol Genet 5:1977-1987 